02.3

\title{
Пространственное распределение каналируемых ионов и пробеги изотопов водорода в кристаллическом кремнии и вольфраме
}

\author{
(ㄱ Д.С. Мелузова, П.Ю. Бабенко, А.П. Шергин, А.Н. Зиновьев \\ Физико-технический институт им. А.Ф. Иофрфе РАН, Санкт-Петербург, Россия \\ E-mail: dmeluzova@gmail.com
}

Поступило в Редакцию 12 сентября 2019г.

В окончательной редакции 12 сентября 2019 г.

Принято к публикации 6 декабря 2019г.

\begin{abstract}
Рассчитаны пробеги ионов H, D в кристаллическом $\mathrm{Si}$ и W. Показано, что с ростом энергии ионов распределение пробегов по глубине распадается на две компоненты: одна связана с рассеянием атомов в поверхностных слоях, а другая характеризует частицы, захваченные в канал. Обнаружено новое явление: после прохождения небольшого расстояния формируется устойчивая пространственная структура компоненты пучка, захваченной в канал. При торможении ионов начинается переход частиц в соседние каналы, и вблизи точки остановки пространственная структура пучка частиц, захваченных в канал, разрушается. Предложена схема эксперимента, позволяющая связать полученное пространственное распределение с угловыми распределениями вылетевших частиц.
\end{abstract}

Ключевые слова: каналирование, пространственное распределение, кристалл, пробеги.

DOI: 10.21883/PJTF.2020.05.49106.18034

Эффект каналирования был открыт Робинсоном и Оеном в 1963 г. [1] в результате численного моделирования проникновения ионов в кристалл. Теория каналирования два года спустя была разработана Линдхардом [2]. В СССР значительный вклад в изучение каналирования и других ориентационных эффектов внес Тулинов [3].

Подробное описание изучения каналирования и областей применения данного эффекта представлено в обзоре [4]. Результаты расчетов и экспериментов по измерению пробегов каналированных атомов водорода в кремнии можно найти в [5-7]. Моделирование каналирования релятивистских протонов было рассмотрено в $[8,9]$. В [10] анализируется точность существующих методов моделирования каналирования.

Задачей настоящей работы является исследование формирования пространственного распределения каналируемых ионов в кристалле и его трансформации при прохождении через кристалл.

В качестве объекта исследования мы выбрали торможение атомов водорода и дейтерия в кристаллическом кремнии и вольфраме. Выбор кремния был обусловлен наличием экспериментальных данных о пробегах и их распределении по глубине, что позволяет проверить точность разработанных нами методов расчета. В случае кристаллического вольфрама экспериментальные данные отсутствуют. Выбор поверхности W(100) обусловлен тем, что кристалл вольфрама обладает простой кристаллографической структурой - кубической объемно центрированной, а большое различие в массах налетающей частицы и атомов мишени, по нашему мнению, должно было способствовать формированию пространственного распределения компоненты частиц, захваченных в канал. Данное явление, а именно формирование пространственного распределения каналируемых частиц, ранее не изучалось.

Численное моделирование проводилось с помощью расчета траекторий частиц [11] с использованием потенциала, полученного в результате применения метода функционала плотности (DFT) [12]. Поверхность кристалла подвергалась бомбардировке моноэнергетическим пучком, и для каждой имплантированной частицы фиксировался ее проективный пробег.

Для проверки правильности получаемых результатов была выбрана система $\mathrm{H}-\mathrm{Si}(100)$, для которой имеются экспериментальные данные о пробегах. На рис. 1, $a$ представлены зависимости среднего проективного пробега от энергии налетающих частиц для системы $\mathrm{H}-\mathrm{Si}(100)$ в случае перпендикулярного падения налетающих частиц и в случае отклонения пучка на $7^{\circ}$ от оси кристалла. Как видно из рисунка, расчет методом траекторий с использованием DFT-потенциала согласуется с экспериментальными данными из работы [7].

Для случая бомбардировки поверхности W(100) атомами D были рассчитаны проективные пробеги и их распределение по глубине материала при разных значениях энергии налетающих частиц и для различной ориентации пучка относительно поверхности. На рис. 1, $b$ представлены распределения пробегов по глубине материала для системы D-W(100) в случае ориентации пучка перпендикулярно поверхности для разных энергий налетающих частиц. Как видно из рисунка, с ростом энергии ионов распределение пробегов по глубине распадается на две компоненты: одна связана со случайным рассеянием атомов в поверхностных слоях, а другая характеризует частицы, захваченные в канал. Данное расслоение наблюдалось экспериментально для системы $\mathrm{K}-\mathrm{W}(111)$ [13]. В исследованном нами случае рассеяния 

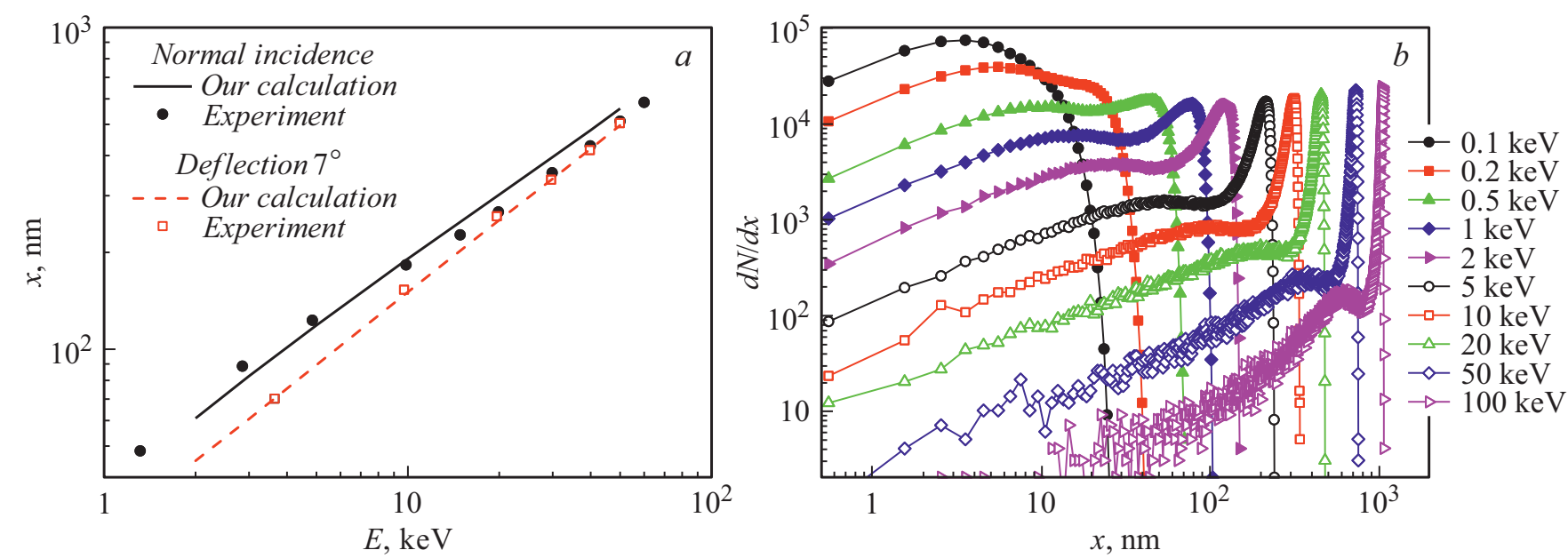

Рис. 1. $a-$ зависимость среднего проективного пробега $x$ от энергии налетающих частиц $E$ для системы $\mathrm{H}-\mathrm{Si}(100)$ в случае перпендикулярного падения пучка (сплошная линия) и в случае отклонения на $7^{\circ}$ (штриховая). Линия - расчет, точки экспериментальные данные [7]. $b$ - распределения пробегов по глубине материала для системы $\mathrm{D}-\mathrm{W}(100)$ в случае ориентации пучка под углом $0^{\circ}$ к нормали при энергии налетающих частиц в диапазоне $0.1-100 \mathrm{keV}$.

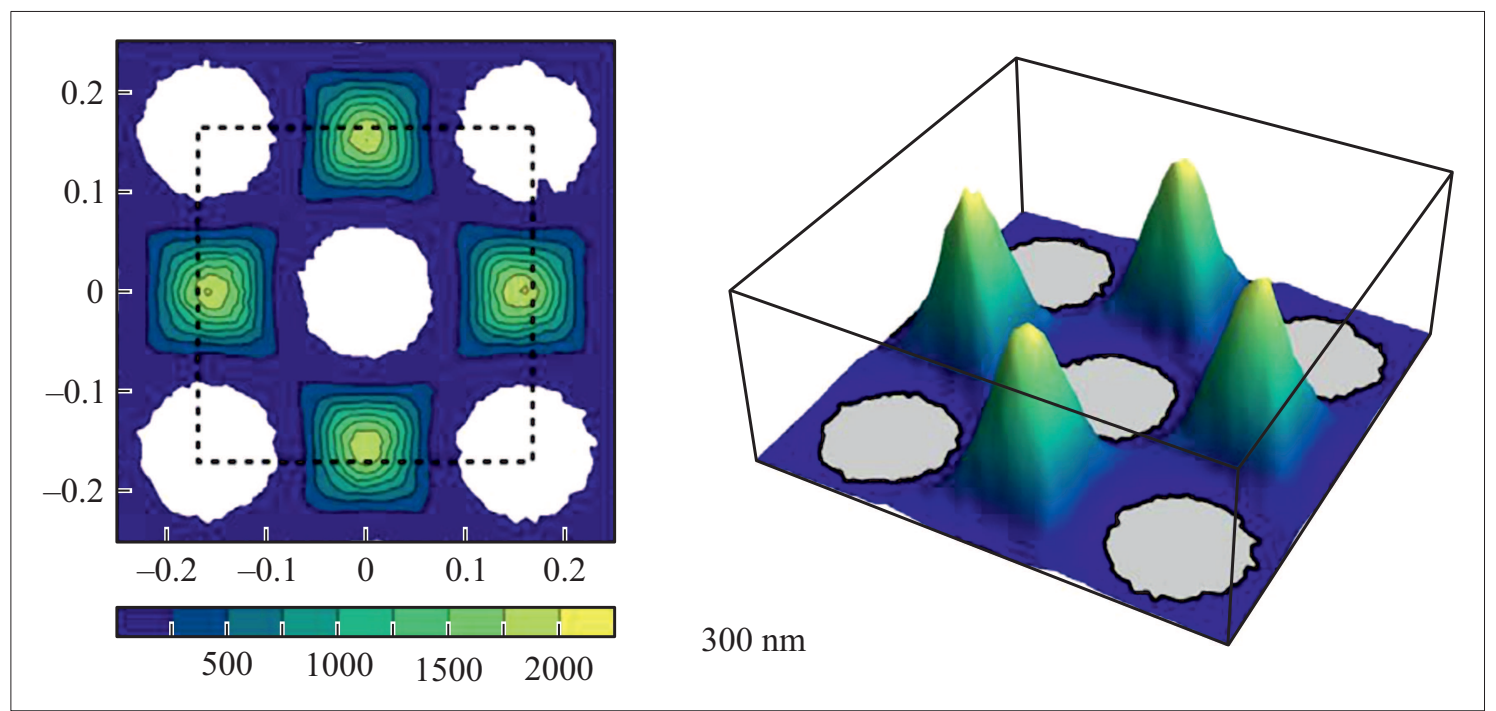

Рис. 2. Пространственное распределение атомов D на глубине $300 \mathrm{~nm}$ в $\mathrm{W}(100)$, начальная энергия налетающих частиц $100 \mathrm{keV}$. На осях - расстояние (в $\mathrm{nm}$ ). Шкала оттенков серого (цветовая шкала в электронной версии статьи) показывает количество частиц, зарегистрированных в каждой точке пространства. Пунктирный квадрат — область облучения пучком D.

легкой частицы данное явление проявляется более ярко и компоненты лучше разделены (рис. $1, b$ ).

С целью проверки правильности подобной интерпретации были выполнены расчеты распределения пробегов по глубине для системы D-W(100) при разных углах падения налетающих частиц, отсчитываемых от нормали к поверхности кристалла. При отклонении направления пучка от кристаллической оси на $0-6^{\circ}$ происходит перестройка распределения пробегов частиц по глубине, при этом уменьшается доля частиц, захваченных в канал.

Расчеты, проводимые с помощью нашего кода, дают возможность получить пространственное распределение налетающих частиц на любой глубине. На рис. 2 пред- ставлено пространственное распределение частиц на глубине $300 \mathrm{~nm}$ для системы D-W(100) при энергии налетающих частиц $100 \mathrm{keV}$. Пучком атомов D была засвечена одна элементарная ячейка площадью $(2 d)^{2}$ (где $d=0.158 \mathrm{~nm})$, показанная на рис. 2 пунктирным квадратом. Шкала оттенков серого (цветовая шкала в электронной версии статьи) показывает количество частиц, зарегистрированных в каждой точке пространства. Расчет проводился для $10^{6}$ налетающих частиц, равномерно заселяющих выделенную площадку. В расчете учитывались тепловые колебания атомов решетки. Амплитуда колебаний принималась равной $0.005 \mathrm{~nm}$. Интересным фактом явилось обнаружение возникновения 

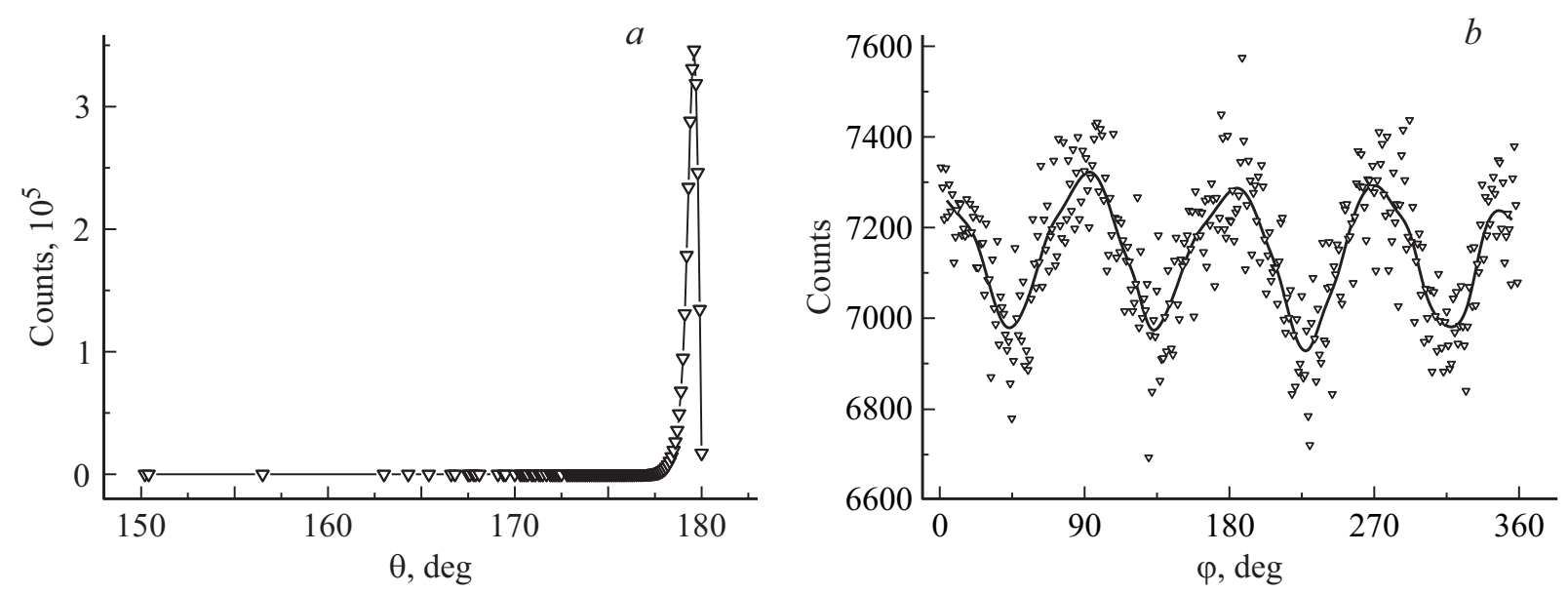

Рис. 3. Распределения вылетевших частиц по углу $\theta$ (угол относительно нормали к поверхности) $(a)$ и азимутальному углу $\varphi(b)$. Толщина мишени $300 \mathrm{~nm}$, начальная энергия налетающих частиц $100 \mathrm{keV}$.

четкого пространственного распределения сформированного пучка каналируемых частиц. Как видно из рис. 2 , при прохождении через кристалл налетающие атомы равномерно распределяются по каналам и образуют четкую пространственную структуру. Ранее этот эффект наблюдался при моделировании каналирования в системе $\mathrm{H}-\mathrm{Si}(111)$ для энергии протонов $2 \mathrm{MeV}$ [14]. Изучаемая нами система имеет другую кристаллическую структуру, и моделирование проводилось для диапазона существенно меньших энергий $(0.1-100 \mathrm{keV})$.

Для того чтобы проследить эволюцию пространственной картины для одного канала, было проведено аналогичное моделирование с засвечиванием более узкой области размером $d^{2}$. Расчет проводился для $2.5 \cdot 10^{5}$ налетающих частиц. На начальном этапе при прохождении $100 \mathrm{~nm}$ захваченные в канал частицы образуют характерное пространственное распределение пирамидальной формы с ориентацией углов „пирамиды“ вдоль потенциальных долин. При начальной энергии частиц $100 \mathrm{keV}$ пробег в вольфраме составляет около $1000 \mathrm{~nm}$. При прохождении пучка до расстояния $700 \mathrm{~nm}$ пространственное распределение частиц сохраняется. На глубине 900 nm наблюдается интенсивное перетекание частиц в соседние каналы. Вблизи точки остановки частиц пространственная структура пучка, захваченного в канал, разрушается.

Образование четкого пространственного распределения пучка каналированных частиц, на наш взгляд, обусловлено многократностью соударений частиц пучка с атомами решетки, которые имеют случайные положения в пространстве вследствие учета тепловых колебаний.

Для проверки полученного результата можно предложить следующий эксперимент. Начальная энергия частиц и толщина мишени выбираются таким образом, чтобы вылет из мишени происходил, когда пространственное распределение каналируемых частиц сформировалось, но еще не разрушилось. Например, можно выбрать толщину мишени $300 \mathrm{~nm}$ и начальную энер- гию налетающих частиц $100 \mathrm{keV}$. Для этих условий на рис. 3, $a, b$ представлены распределения вылетевших частиц по углу $\theta$ (угол относительно нормали к поверхности) и азимутальному углу $\varphi$. Распределение по углу $\theta$ позволит получить информацию о распределении потенциала в канале, а распределение по углу $\varphi$ отражает строение кристалла и может быть использовано для анализа топографии поверхностного слоя пленки.

Если дополнить эксперимент анализом энергетического спектра вылетевших частиц, можно получить интересную информацию о величинах электронных тормозных потерь при движении частицы в канале.

Сформулируем основные выводы.

1. Рассчитаны пробеги ионов водорода в $\mathrm{Si}(100)$ при перпендикулярном падении пучка на поверхность и при отклонении от нормали. Результаты обоих расчетов находятся в хорошем согласии с экспериментом.

2. В результате расчета пробегов атомов дейтерия в W(100) было показано, что с ростом энергии ионов распределение пробегов по глубине распадается на две компоненты, одна из которых связана со случайным рассеянием атомов в поверхностных слоях, а другая характеризует частицы, движущиеся по каналу.

3. Обнаружено образование устойчивой пространственной структуры пучка частиц, захваченных в канал, и исследована зависимость этого пространственного распределения от пройденного расстояния. Показано, что пространственная структура сохраняется вплоть до расстояний, составляющих до $90 \%$ от пробега частиц.

4. Предложена схема эксперимента по анализу топографии кристалла и определению характеристик каналирования на основе анализа угловых и энергетических распределений вылетевших частиц.

\section{Финансирование работы}

Работа выполнена в рамках государственного задания Министерства образования и науки РФ для Федераль- 
ного государственного бюджетного учреждения науки Физико-технического института им. А.Ф. Иоффе Российской академии наук.

\section{Конфликт интересов}

Авторы заявляют, что у них нет конфликта интересов.

\section{Список литературы}

[1] Robinson M.T., Oen O.S. // Appl. Phys. Lett. 1963. V. 2. P. 30 32. DOI: $10.1063 / 1.1753757$

[2] Lindhard J. // Kgl. Dan. Viden. Sels. Mat.-Fys. Medd. 1965. V. 14. P. 34.

[3] Тулинов А.Ф. // УФН. 1967. Т. 87. № 12. С. 585-598. DOI: $10.3367 /$ UFNr.0087.196512a.0585

[4] Vantomme A. // Nucl. Instrum. Meth. Phys. Res. B. 2016. V. 371. P. 12-26. DOI: 10.1016/j.nimb.2015.11.035

[5] Ćosić M., Nešković N., Petrović S. // Nucl. Instrum. Meth. Phys. Res. B. 2019. V. 444. P. 10-22. DOI: $10.1016 /$ j.nimb.2019.02.004

[6] Hobler G., Bourdelle K.K., Akatsu T. // Nucl. Instrum. Meth. Phys. Res. B. 2006. V. 242. P. 617-619.

DOI: $10.1016 /$ j.nimb.2005.08.181

[7] Ligeon E., Guivarc'h A. // Rad. Eff. 1976. V. 27. P. 129-137. DOI: $10.1080 / 00337577608243025$

[8] Кощеев В.П., Моргун Д.А., Штанов Ю.Н. // Письма в ЖТФ. 2012. Т. 38. В. 12. C. 87-94.

[9] Комеев В.П., Моргун Д.А., Штанов Ю.Н. // Письма в ЖТФ. 2013. Т. 39. В. 20. C. 77-86.

[10] Hobler G., Nordlund K. // Nucl. Instrum. Meth. Phys. Res. B. 2019. V. 449. P. 17-21. DOI: 10.1016/j.nimb.2019.04.029

[11] Мелузова Д.С., Бабенко П.Ю., Шергин А.П., Зиновьев А.Н. // Поверхность. 2019. Т. 4. С. 74-78. DOI: $10.1134 / \mathrm{S} 0207352819040127$

[12] Zinoviev A.N., Norlund K. // Nucl. Instrum. Meth. Phys. Res. B. 2017. V. 406. P. 511-517. DOI: 10.1016/j.nimb.2017.03.047

[13] Мейер Д., Эриксон Л., Девис Д. Ионное легирование полупроводников. М.: Мир, 1973. С. 59.

[14] Motapothula M.R., Breese M.B.H. // Eur. Phys. J. B. 2018. V. 91. P. 49. DOI: $10.1140 / \mathrm{epjb} / \mathrm{e} 2018-80580-4$ 